\title{
Determination of Flaw Size from Thermographic Data
}

\author{
William P. Winfree*, Patricia A. Howell ${ }^{\dagger}$ and Joseph N. Zalameda ${ }^{\dagger}$ \\ * Mail Stop 225, NASA Langley Research Center, Hampton, VA 23681-0001 \\ ${ }^{\dagger}$ Mail Stop 231, NASA Langley Research Center, Hampton, VA 23681-0001
}

\begin{abstract}
Conventional methods for reducing the pulsed thermographic responses of delaminations tend to overestimate the size of the flaw. Since the heat diffuses in the plane parallel to the surface, the resulting temperature profile over the flaw is larger than the flaw. A variational method is presented for reducing the thermographic data to produce an estimated size for the flaw that is much closer to the true size of the flaw. The size is determined from the spatial thermal response of the exterior surface above the flaw and a constraint on the length of the contour surrounding the flaw. The technique is applied to experimental data acquired on a flat bottom hole composite specimen.
\end{abstract}

Keywords: Thermography,Composites,Flaw Characterization

PACS: $<44.10 .+\mathrm{i}>$

\section{INTRODUCTION}

An effective method of rapid inspection of large carbon fiber reinforced polymer (CFRP) composite structures is flash thermography. The increased utilization of composites in commercial and military aircraft increases the need for viable inspection techniques for ensuring aircraft safety and reliability. Thermography is effective for detection of fatigue and impact damage in CFRP composites[1, 2, 3, 4, 5, 6]. A variety of data reduction techniques have been shown to improve flaw detection[7, 8]. To assess the significance of the damage, it is often essential to be able to characterize the location and size of the damage. Previous efforts to estimate the depth of the damage have focused on the time evolution of the thermal response[7, 9, 10]. Since the heat diffuses in a direction parallel to the surface as the thermal disturbance from a flaw diffuses to the surface, the size of the thermal response is larger than the size of the flaw. Methods for obtaining the correct flaw size from the thermal response have included estimating the size from the time evolution of the shape of the thermal response[11, 12], from the gradient of the thermal response[13] and a deconvolution of the point spread function of the thermal response[14, 15].

The "deblurring" of the thermal response to compensate for the diffusion in the plane parallel to the surface is similar to the deblurring problem in image processing. An effective technique for deblurring in image processing is a variational method, where a blurred estimation for the true image is compared to the measured image. The variational method for image processing is well developed[16, 17]. The direct application of image processing deblurring techniques to deblurring of thermographic images is somewhat limited since the desired flaw shapes are binary images (ie. either a flaw exists at a particular location or it does not), rather than gray or color images as is the case for image processing. This paper therefore examines of variational approach where the thermal response is a "blurred" image of a binary image. The method is formulated in a manner similar to the image segmentation technique developed by Chan and Vese[18].

A simple way to fabricate and characterize a flaw in a specimen is a flat bottom hole. While it does not accurately represent a delamination, a flat bottom hole specimen was used to test the data reduction technique. The solution of the variational problem requires iterative solutions to a partial differential equation. The variational method is easy to formulate if the blurring function is a Gaussian, therefore a simple model for the thermal response of a flat bottom hole based on the convolution of a Gaussian kernal is presented and compared to a finite element simulation of the thermal response in the following section. 


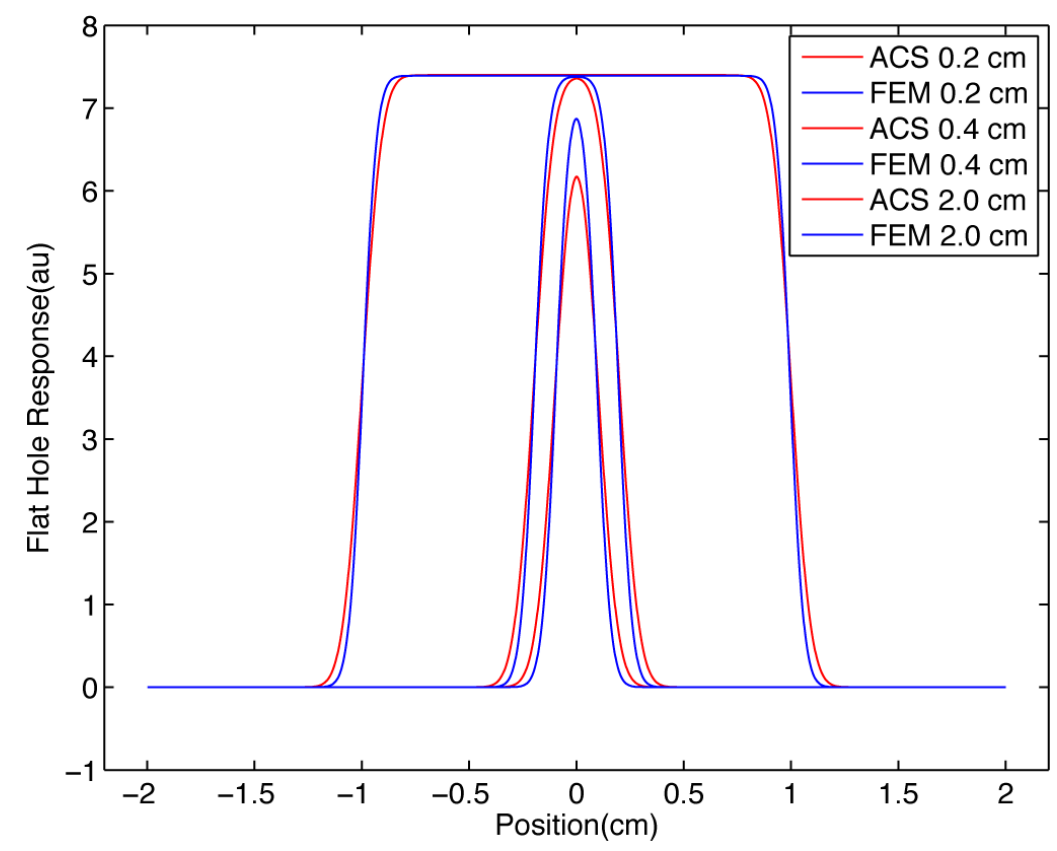

FIGURE 1. Comparison of the approximate convolution solution to finite element results for slots $0.2 \mathrm{~cm}, 0.4 \mathrm{~cm}$ and $2.0 \mathrm{~cm}$ wide and $0.05 \mathrm{~cm}$ below the surface. Compared profiles are for 0.5 seconds following the instantaneous surface heating. The approximate convolution solutions based on Eq.(2) are labeled as ACS and the finite element solutions are labeled as FEM. The nominal response (no flaw) has been subtracted from the thermal responses leaving only the responses due to the slot.

\section{THERMAL RESPONSE OF FLAT BOTTOM HOLE}

\section{Approximate Analytical Solution for Flat Bottom Hole}

The thermal response to an instantaneous flux at one surface of a single layer is well known. The first two terms of the series solution of early time response based on image sources are

$$
T(t)=\frac{F \sqrt{\kappa}}{K \pi \sqrt{t}}\left(1+2 e^{-\frac{l^{2}}{\kappa t}}\right)
$$

where $K$ is the thermal conductivity, $\kappa$ is the thermal diffusivity, $l$ is the thickness of the layer and $F$ is the instantaneous flux applied to the front surface. A possible approximate solution for a flat bottom hole response can be found by placing two sources, one below and one above the front surface, at a distance equal to twice the depth of the top of the flat bottom hole with the shape of the flat bottom hole. The thermal response at the front surface is then given by

$$
T(t)=\frac{F \sqrt{\kappa}}{K \pi \sqrt{t}}\left(1+2 e^{-\frac{l^{2}}{\kappa t}} \frac{1}{4 \pi \kappa t} \iint_{\Omega} u\left(x^{\prime}, y^{\prime}\right) e^{\frac{-\left(x-x^{\prime}\right)^{2}+\left(y-y^{\prime}\right)^{2}}{4 \kappa t}} d x^{\prime} d y^{\prime}\right),
$$

where $u\left(x^{\prime}, y^{\prime}\right)$ is the shape of the top surface of the flat bottom hole. If $u\left(x^{\prime}, y^{\prime}\right)$ is a plane (that is $\left.u\left(x^{\prime}, y^{\prime}\right)=1\right)$, then

$$
\int_{-\infty}^{\infty} \int_{-\infty}^{\infty} e^{\frac{-\left(x-x^{\prime}\right)^{2}+\left(y-y^{\prime}\right)^{2}}{4 \kappa t}} d x^{\prime} d y^{\prime}=4 \pi \kappa t
$$

and Eq.(2) reduces to Eq.(1) the solution for a layer with a thickness equal to the depth of the plane.

To assess the accuracy of this approximation, the thermal response as calculated from Eq.(2) was compared to the results of a finite element calculation for a long slot in a layer. The material properties of the layer were a thermal conductivity of $0.97 \mathrm{~W} / \mathrm{m} /{ }^{\circ} \mathrm{K}$, specific heat of $1200 \mathrm{~J} / \mathrm{kg} /{ }^{\circ} \mathrm{K}$ and a density of $1600 \mathrm{~kg} / \mathrm{m}^{3}$. These are values commonly used for the surface normal thermal properties of a composite. A comparison of the finite element results and the thermal profiles obtained for $0.05 \mathrm{~cm}$ deep slot in a plate $0.2 \mathrm{~cm}$ thick from Eq.(2) is shown in Fig. 1. As can be seen 


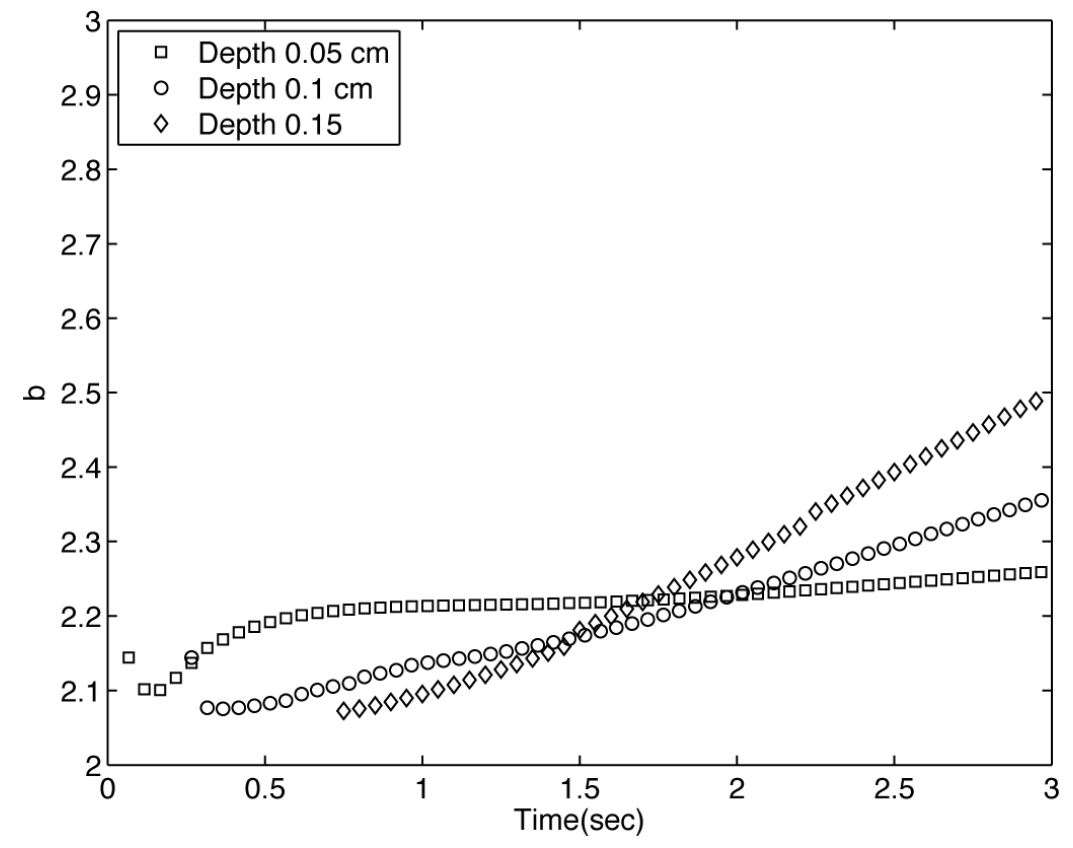

FIGURE 2. Calculated the values of $b$ as determined from finite element simulations of a $2 \mathrm{~cm}$ wide slot at three different depths in a $0.2 \mathrm{~cm}$ thick plate.

from that figure, for large flaws near the surface where the size of the slot is significantly larger than the effective diffusion length $\left(2(\kappa t)^{1 / 2}\right)$ ), the maximum amplitude both the finite element simulation and the convolution are the same. Near the edges of the slot, the convolution approximation produces more spreading of the thermal response than the finite element simulation, suggesting the $4 \kappa t$ in the Gaussian convolution kernel is too large.

\section{Thermal Response Based on Finite Element Simulation of Slot in Plate}

An important attribute of Eq.(2) is the in-plane diffusion of the response is not dependent on the depth of the flat bottom hole, but only on the time of the measured response. This implies that two holes at different depths, but having the same shape, will have the same shape thermal responses at the surface at any given time. While the shapes are the same, the amplitudes of the responses will be different and depend on depth. Since Eq.(2) seems to give a reasonable approximate solution, finite element simulations were performed for $2 \mathrm{~cm}$ wide slots with top surfaces $0.05 \mathrm{~cm}, 0.1$ $\mathrm{cm}$ and $0.15 \mathrm{~cm}$ below the surface in a $0.2 \mathrm{~cm}$ thick plate. From these results an empirical approximation was sought.

The empirical approximation assumes the early time thermal response has a form similar to Eq.(2)

$$
T(t)=\frac{F \sqrt{\kappa_{z z}}}{K \pi \sqrt{t}}\left(1+2 e^{-\frac{l^{2}}{\kappa_{z z} t}} \frac{1}{\pi b \kappa_{x x} t} \iint_{\Omega} u\left(x^{\prime}, y^{\prime}\right) e^{\frac{-\left(x-x^{\prime}\right)^{2}+\left(y-y^{\prime}\right)^{2}}{b \kappa_{x x} t}} d x^{\prime} d y^{\prime}\right),
$$

where $b$ is a constant. The constant $b$ can be determined by comparison of the calculation of the thermal response using Eq. (4) and the results of the finite element simulations where the finite element simulated thermal response of the slot is larger than the computational accuracy of the simulation. For a slot, the shape of thermal response as determined from Eq.(4) is

$$
T(t)=\frac{F \sqrt{\kappa_{z z}}}{K \pi \sqrt{t}}\left(1+e^{-\frac{l^{2}}{\kappa_{z z} t}}\left(\operatorname{erf}\left(\frac{(x-x 1)}{\sqrt{b \kappa_{x x} t}}\right)-\operatorname{erf}\left(\frac{(x-x 2)}{\sqrt{b \kappa_{x x} t}}\right)\right)\right),
$$

where $x 1$ and $x 2$ are the positions of the left side and right side of the slots respectively. Since $x 1$ and $x 2$ are known, the thermal responses calculated from the finite element simulation can be fitted to determine $b$. The calculated values for $b$ for each time step of the finite element simulation are shown in Fig. 2. The value of $b$, as determined from fitting the finite element simulations, vary about 2.2 instead of a value of 4 , the effective value of $b$ in Eq.(2). 


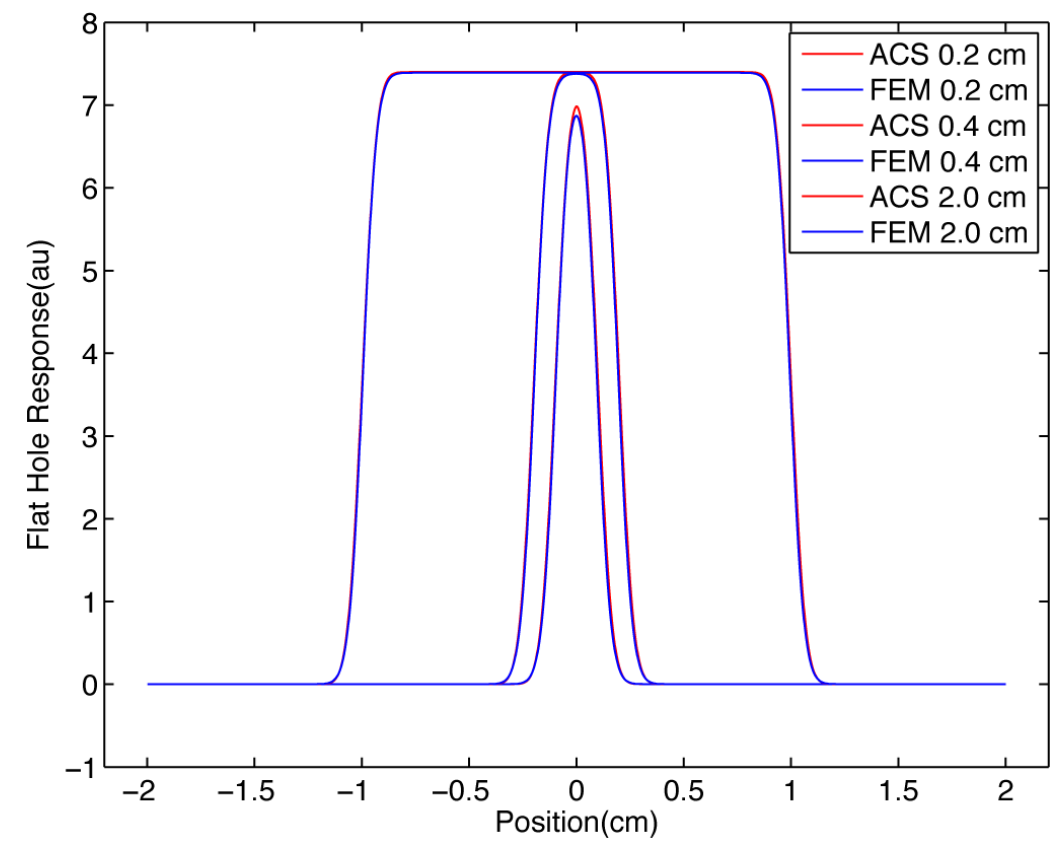

FIGURE 3. Comparison of the approximate convolution solution to finite element results for slots of $0.2 \mathrm{~cm}, 0.4 \mathrm{~cm}$ and 2.0 $\mathrm{cm}$ wide and $0.05 \mathrm{~cm}$ below the surface. Compared profiles are for 0.5 seconds following the instantaneous surface heating. The approximate convolution solutions based on Eq.(2) are labeled as ACS and the finite element solutions are labeled as FEM.The nominal solution has been subtracted from the total solutions leaving only the response due to the slot.

Using a value of 2.2 for $b$ in Eq.(4), the thermal responses were calculated for the slotted plate configurations discussed in the previous section and the results are compared with the finite element solutions in Fig. 3. As can be seen from a comparison of that figure and Fig. 1, Eq. (4) gives a better approximation of the thermal response than Eq.(2) when $b$ is equal to 2.2. In particular it gives a better approximation near the edges of the slot. For all but the smallest slot, it is difficult to distinguish the finite element simulation results from the convolution results. Comparisons of the finite element simulations for deeper slots, $(0.1 \mathrm{~cm}$ and $0.15 \mathrm{~cm}$ below surface) give similar results.

In the following section this approximation for the thermal response is applied within a variational method formulation to estimate the size of the top of flat bottom holes.

\section{APPLICATION OF VARIATIONAL PRINCIPLE TO FLAW SIZING}

The integral equation representation of the variational principle in image processing is

$$
E(p(x, y))=\iint_{\Omega} F(p(x, y), d(x, y)) d x d y+\gamma g(p(x, y)),
$$

where $E(p(x, y))$ is the energy to be minimized, $p(x, y)$ is the desired image, $d(x, y)$ is the acquired image, $F(p, d)$ is the relationship between the acquired image and the desired image, $g(p)$ is a constraint and $\gamma$ is a constant.

For sizing of a flat bottom hole from a single thermographic image that occurs at time $\tau$, if one assumes the signal due to the flat bottom hole is given by $D(u(x, y), x, y, \tau)$ where $u(x, y)$ is the shape of the flat bottom hole, then a possible energy to be minimized is

$$
E(u(x, y))=\iint_{\Omega}\left[(D(u(x, y), x, y, \tau)-T(x, y, \tau))^{2}+\gamma \delta(\phi(x, y))|\nabla u(x, y)|\right] d x d y,
$$

where $T(x, y, \tau)$ is the measured thermal response at time $\tau$ and the constraint is proportional to the circumference of the flaw. To simplify estimating the shape of the flat bottom hole, rather than solving for $u(x, y)$ directly, a method is used that is similar to that proposed for image segmentation by Chan and Vese[18], that is the outer boundary of the 
shape is represented by the zero level set of a Lipschitz function $\phi(x, y)$. The shape of the top of the flat bottom hole is given by $H(\phi(x, y))$, where $H$ is the heavy side function.

To simplify the analysis, at each time step the nominal response of the plate is subtracted from the simulation of the slot in the plate leaving the response from the slot. This is normally considered the contrast in the image, with the nominal response set to zero. The response of the flat bottom hole at a given time $\tau$ is

$$
R(\phi(x, y))=A(l, \tau) \frac{1}{\pi B} \iint_{\Omega} H\left(\phi\left(x^{\prime}, y^{\prime}\right)\right) e^{\frac{-\left(x-x^{\prime}\right)^{2}+\left(y-y^{\prime}\right)^{2}}{B}} d x^{\prime} d y^{\prime}
$$

where $B=b \kappa t$ and $A(l, \tau)$, the amplitude of the response, is dependent only on the depth of the top of the flat bottom hole $(l)$ and time of the response and shows explicitly that the response shape is independent of the depth of the top of the flat bottom hole. To first order, $A(l, \tau)$ is given by

$$
A(l, \tau)=2 e^{-\frac{l^{2}}{\kappa \tau}}
$$

a value that is found when reducing the data and could be used to estimate the depth of the top of the flat bottom hole. Representing $R(\phi(x, y))$ as $G * H(\phi(x, y))$, then Eq.(7) becomes

$$
E^{\prime}(\phi(x, y))=\iint_{\Omega}\left[(G * H(\phi(x, y))-T(x, y, \tau))^{2}+\gamma \delta(\phi(x, y))|\nabla H(\phi(x, y))|\right] d x d y .
$$

Following the approach taken by Chan and Vese[18], an approximation is substituted for the heavy side function and the $\phi(x, y)$ is determined from associated Euler-Lagrange equation

$$
\delta_{\varepsilon}(\phi(x, y))\left(G *\left(G * H_{\varepsilon}(\phi(x, y))-T(x, y, \tau)\right)+\gamma \operatorname{div}\left(\frac{\nabla \phi(x, y)}{|\nabla \phi(x, y)|}\right),=0\right.
$$

where we have chosen a slightly different approximation for the heavy side function of

$$
H_{\varepsilon}(x)=(1+\operatorname{erf}(x / \varepsilon)) / 2
$$

and it's derivative

$$
\delta_{\varepsilon}(x)=\frac{e^{-x^{2} / \varepsilon^{2}}}{\sqrt{\pi} \varepsilon} .
$$

$\varepsilon=1 / 2$ seems to yield relatively good results for the spatial resolution of the thermographic images of interest. Assuming an initial guess for the boundary of the upper surface of the flat bottom hole is a single large circle, and the initial guess for $\phi(x, y)$ is a signed distance function from that boundary, the solution to Eq.(11) is found numerically using an iterative finite difference scheme in a manner similar to [18] and [19].

\section{SYSTEM FOR MEASUREMENT OF THERMAL RESPONSE}

The thermal response of the composite specimen was performed with a commercial flash thermographic measurement system $[4,7]$. The front surface of the composite is heated with flash lamp. The flash duration has been measured to be approximately 0.008 second. Since the earliest thermal responses of interest occur approximately one tenth of a second after an instantaneous heat pulse, this is a good estimate of an impulse excitation. The thermal response was measured with a focal plane array infrared imager detector with an array size of 640x512 that operates in the 3-5 micrometer wavelength band. The approximate spatial resolution of the acquired thermography images was $0.04 \mathrm{~cm}$. The imager digital output frame rate was 60 hertz and was connected to a real time digital image processor to acquire the output images. All of the data of interest was collected within the 2.5 seconds after the flash heating.

\section{COMPOSITE FLAT BOTTOM HOLE SPECIMEN}

The composite specimen was approximately $10 \mathrm{~cm}$ x $10 \mathrm{~cm}$ with at thickness of $0.212 \mathrm{~cm}$. Nine approximate flat bottom holes were drilled in from the back side of the specimen with depths of approximately one fourth, one half 


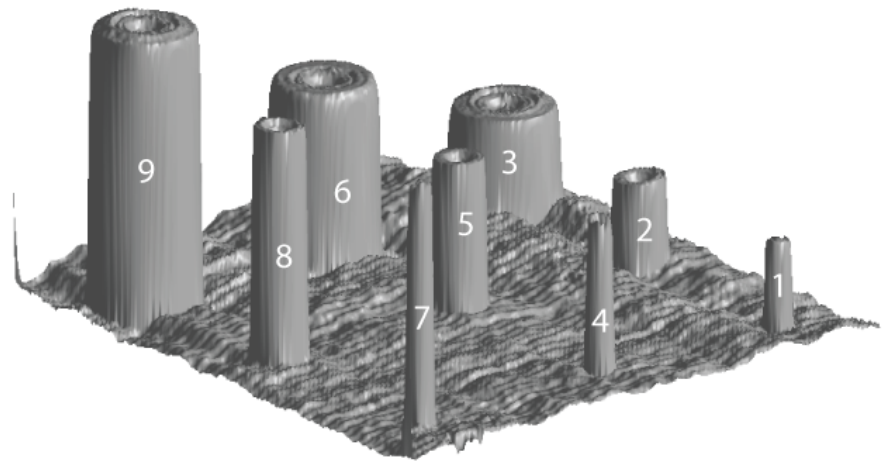

(a)

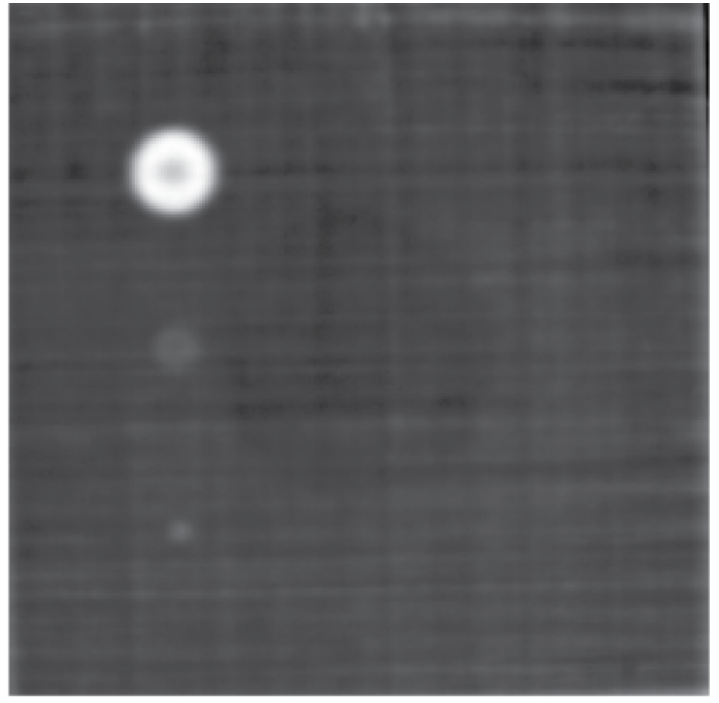

(b)

FIGURE 4. Computed tomography and thermal response of composite specimen. a. Representation of the x-ray computed tomography data acquired on composite flat bottom hole specimen. The hole numbers used to identify the holes in the paper are superimposed on the image. b. The measured thermal response from specimen acquired 0.25 seconds after the flash heating.

TABLE 1. Size and Depth of Holes in Composite Flat Bottom Hole Specimen Based on the Computed Tomography Data

\begin{tabular}{cccc}
\hline $\begin{array}{c}\text { Hole } \\
\text { Number }\end{array}$ & $\begin{array}{c}\text { Depth from } \\
\text { front surface }\end{array}$ & $\begin{array}{c}\text { Percent thickness } \\
\text { above hole }\end{array}$ & Diameter \\
\hline 1 & $0.154 \pm 0.003 \mathrm{~cm}$ & $73 \%$ & $0.32 \pm 0.01 \mathrm{~cm}$ \\
2 & $0.156 \pm 0.003 \mathrm{~cm}$ & $74 \%$ & $0.64 \pm 0.01 \mathrm{~cm}$ \\
3 & $0.148 \pm 0.005 \mathrm{~cm}$ & $70 \%$ & $1.28 \pm 0.01 \mathrm{~cm}$ \\
4 & $0.105 \pm 0.008 \mathrm{~cm}$ & $50 \%$ & $0.32 \pm 0.01 \mathrm{~cm}$ \\
5 & $0.109 \pm 0.004 \mathrm{~cm}$ & $51 \%$ & $0.64 \pm 0.01 \mathrm{~cm}$ \\
6 & $0.097 \pm 0.008 \mathrm{~cm}$ & $46 \%$ & $1.29 \pm 0.01 \mathrm{~cm}$ \\
7 & $0.051 \pm 0.019 \mathrm{~cm}$ & $24 \%$ & $0.32 \pm 0.01 \mathrm{~cm}$ \\
8 & $0.053 \pm 0.007 \mathrm{~cm}$ & $25 \%$ & $0.64 \pm 0.01 \mathrm{~cm}$ \\
9 & $0.027 \pm 0.014 \mathrm{~cm}$ & $13 \%$ & $1.28 \pm 0.01 \mathrm{~cm}$ \\
\hline
\end{tabular}

and three fourths of the thickness, and diameters of approximately $1.27 \mathrm{~cm}, 0.63 \mathrm{~cm}$ and $0.32 \mathrm{~cm}$. A more accurate measurements of the depth and diameters were determined from x-ray computed tomography data shown in Fig. 4 and are given in Table 1. For comparison, an early time thermal response is shown in the same figure.

As can be seen from the computed tomography data in Fig.4, the top of the flat bottom holes are not quite flat. From an examination of the standard deviations of the depth, the holes with a diameter of approximately $0.64 \mathrm{~cm}$ have the flattest tops and the largest holes have the least flat tops. The deeper the hole was drilled the less flat the top of the hole. This lack of flatness is reflected in the thermal response shown as Fig.4. In the thermal image it is clear that the slight increase in material at the center of the largest hole results in a slightly cooler location at the center of the hole response. Measurements at later times have this same cool spot at the center of all the largest holes, however, it is not as significant in the shallower the holes. 


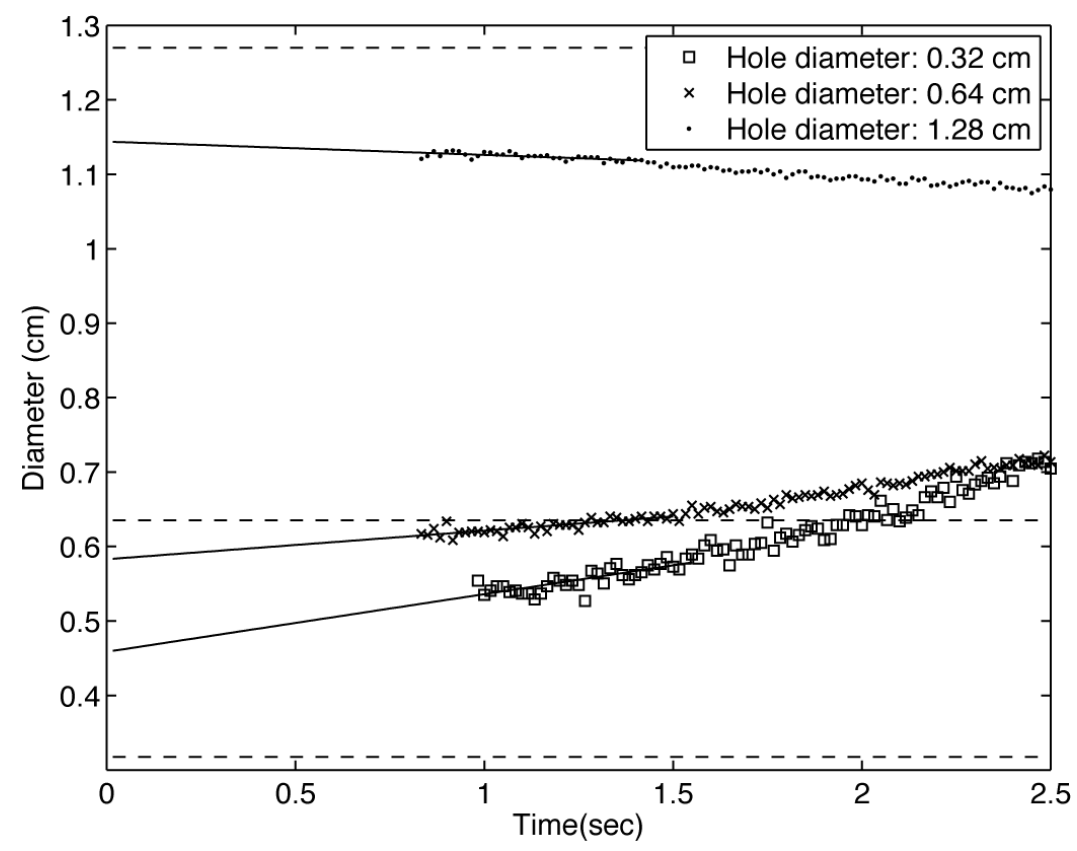

FIGURE 5. The estimation of the diameter of the thermal response of flat bottom holes with tops approximately $0.1 \mathrm{~cm}$ beneath the surface based on the reduction of images acquired at different times. The linear fits of the early time values used to estimate the diameters of the flat bottom holes are also shown in the the figure as solid lines and the nominal diameters of the holes are shown as dashed lines.

\section{ESTIMATION OF DIAMETER OF FLAT BOTTOM HOLE FROM THERMOGRAPHIC RESPONSE}

Two methods were used to estimate the diameters of the flat bottom hole specimens. Both of the methods have the advantage of not requiring an estimation of the depth of the flaw. The first method was based on a technique presented by Almond and Lau[11]. A region centered on the flat bottom hole $2.44 \mathrm{~cm}$ by $2.44 \mathrm{~cm}$ (61x61 pixels) of the thermal response corresponding to a single flat bottom hole was selected from the data. At each time step where the thermal response of the flat bottom hole was significantly greater than the noise in the image, the nominal and maximum response was determined from the region following Gaussian smoothing. For each time step the data was thresholded with a cutoff at the mean of the maximum and nominal response to give a binary image representing the number of points with amplitudes greater the half the maximum. The sum of the number of pixels above the threshold times the area of a pixel is taken to be the area of the response for a given time step. Assuming the flaw shape is circular, the estimated diameter of the circle is determined from this area. The diameters of the thermal response as measured for the flat bottom holes with the depths of approximately $0.1 \mathrm{~cm}$ are shown in Fig.5. The early time dependence of the the diameter of the response tends to be linear. The diameter of the response at time zero extrapolated from a linear fit of the early time response is the estimated diameter of the flat bottom hole. In Fig.5, the linear fits used to estimate the diameter of the holes are also shown. A difference between the time dependence of the diameter of the thermal response shown here and the results obtained by Almond and Lau is the two smaller holes have positive slopes, while in the paper by Almond and Lau, all of the slopes were negative. It should be noted that Almond and Lau assumed a flaw size that was relatively large compared to the effective diffusion length $\left(2(\kappa t)^{1 / 2}\right.$ ) which is approximately 0.3 $\mathrm{cm}$ for this specimen. The estimated diameters and the associated errors based on the confidence interval of the fit are given in Table .2.

The same data was analyzed with the variational method. For each region of the thermal response around the center of the flat bottom hole, the nominal response at each time step of the composite was subtracted from the data in the region, producing a "contrast" image. Each "contrast" image is input into Eq.(11) as $T(x, y, \tau)$. The variational method gives an estimation of the shape of the hole for each time step. A typical estimated shape obtained for the $0.32 \mathrm{~cm}$ diameter hole, $0.105 \mathrm{~cm}$ below the front surface (hole \#4) based on the data obtained at 1.0 seconds is shown in 
TABLE 2. Size and Depth of Holes in Composite Flat Bottom Hole Specimen Based on the Computed Tomography Data

\begin{tabular}{ccccc}
\hline $\begin{array}{c}\text { Hole } \\
\text { Number }\end{array}$ & $\begin{array}{c}\text { Depth from } \\
\text { front surface }\end{array}$ & $\begin{array}{c}\text { Diameter } \\
\text { of hole }\end{array}$ & $\begin{array}{c}\text { Diameter estimated from full } \\
\text { width at half maximum* }\end{array}$ & $\begin{array}{c}\text { Diameter estimated from } \\
\text { variational analysis }\end{array}$ \\
\hline 1 & $0.154 \pm 0.003 \mathrm{~cm}$ & $0.32 \pm 0.01 \mathrm{~cm}$ & $0.82 \pm 0.09 \mathrm{~cm}$ & $0.4 \pm 0.2$ \\
2 & $0.156 \pm 0.003 \mathrm{~cm}$ & $0.64 \pm 0.01 \mathrm{~cm}$ & $0.58 \pm 0.05 \mathrm{~cm}$ & $0.64 \pm 0.02$ \\
3 & $0.148 \pm 0.005 \mathrm{~cm}$ & $1.28 \pm 0.01 \mathrm{~cm}$ & $1.03 \pm 0.02 \mathrm{~cm}$ & $1.23 \pm 0.007$ \\
4 & $0.105 \pm 0.008 \mathrm{~cm}$ & $0.32 \pm 0.01 \mathrm{~cm}$ & $0.47 \pm 0.02 \mathrm{~cm}$ & $0.34 \pm 0.06 \mathrm{~cm}$ \\
5 & $0.109 \pm 0.004 \mathrm{~cm}$ & $0.64 \pm 0.01 \mathrm{~cm}$ & $0.581 \pm 0.007 \mathrm{~cm}$ & $0.62 \pm 0.01 \mathrm{~cm}$ \\
6 & $0.097 \pm 0.008 \mathrm{~cm}$ & $1.29 \pm 0.01 \mathrm{~cm}$ & $1.144 \pm 0.007 \mathrm{~cm}$ & $1.22 \pm 0.06 \mathrm{~cm}$ \\
7 & $0.051 \pm 0.019 \mathrm{~cm}$ & $0.32 \pm 0.01 \mathrm{~cm}$ & $0.308 \pm 0.003 \mathrm{~cm}$ & $0.37 \pm 0.007 \mathrm{~cm}$ \\
8 & $0.053 \pm 0.007 \mathrm{~cm}$ & $0.64 \pm 0.01 \mathrm{~cm}$ & $0.58 \pm 0.05 \mathrm{~cm}$ & $0.56 \pm 0.02 \mathrm{~cm}$ \\
9 & $0.027 \pm 0.014 \mathrm{~cm}$ & $1.28 \pm 0.01 \mathrm{~cm}$ & $1.168 \pm 0.002 \mathrm{~cm}$ & $1.07 \pm 0.03 \mathrm{~cm}$ \\
\hline
\end{tabular}

* Using method for estimation of size from time response discussed in Almond and Lau[11]

Fig.6. The thermal data used to calculate this estimate, a calculation of the thermal response from the shape of the hole and the difference between the calculated and measured responses are also shown in the figure. As can be seen from the figure, the shape of the thermal response calculated from the estimated shape for the hole agrees with the measured response to within the "noise" floor of the data. The "noise" floor is not random noise, but is a result of the inhomogeneities in thermal response of the composite. Currently this process has not been optimized and requires approximately 20 seconds per image to estimate the shape.

For each time step the shape of the flat bottom hole was estimated using the variational method. The shape is a binary image with ones for the flat bottom holes and zeros where there was no hole. The sum of the number of pixels in the shape of flaw times the area of a pixel is the estimate of the area of the area of the flat bottom hole based on a single time step. The estimated diameter of the flat bottom hole based on a single time step assumes the shape is circular. For each time step the estimated diameters of the flat bottom holes with the depths of approximately $0.1 \mathrm{~cm}$ are shown in Fig.7. As can seen from the figure, all of the time steps give approximately the same diameters. Relatively flat slopes of the time dependencies were also seen for all of the other holes, except hole \#1 $(0.154 \mathrm{~cm}$ below surface and $0.32 \mathrm{~cm}$ in diameter). This is expected if the convolution given in Eq.(4) is a good approximation for the measured thermal response. To obtain the flat time dependency, the diffusivity parallel to the surface $\left(\kappa_{x x}\right)$ was assumed to be approximately $0.025 \mathrm{~cm}^{2} / \mathrm{sec}$ or about 5 times the measured surface normal thermal diffusivity $\left(\kappa_{z z}\right)$. The estimated diameters and the associated errors based on the standard deviation of the measurements at each time step are also given in Table 2 .

The techniques both produce a systematic error when estimating the diameter of the $1.28 \mathrm{~cm}$ holes. For both techniques the estimated diameter is smaller than the true diameter. This is perhaps a result of the top of the hole not being quite flat. It was noted in the discussion of Fig. 4 that the lack of flatness results in lower values at the center of the holes than at the edge for early times in the thermal response, which would not be expected for an ideal fat bottom hole response. This lack of flatness may have resulted in the systematic error of 1 to $2 \mathrm{~mm}$.

For both techniques the estimates for the diameters of the mid size holes were most accurate. For both techniques the estimated diameters were within $0.5 \mathrm{~mm}$ of the diameters measured from the computed tomography data. For the two deeper flaws, where the earliest time steps with contrasts large enough to allow for a measurement, the shape of the thermal response does not have the appearance of flat bottom hole response, but more closely resembles a two dimensional Gaussian as can be seen in Fig.6, the variational technique estimates were slightly better those based on the full width at half maximum.

The variational estimates for the diameters of the smallest size holes were more accurate than the diameters estimated from the full width at half maximum. The full width at half maximum results in estimated diameters that are much larger than the size of the hole for the two deepest 0.32 diameter holes. This is perhaps partly a result of uncertainty in the estimation of the values of the maximums. However, it is also a result of the spreading of the thermal response in the plane parallel to the surface. The variational approach gives an estimate of the diameter that is in excellent agreement with the computed tomography estimate, while the deepest hole is in agreement, but has very large error bars. 


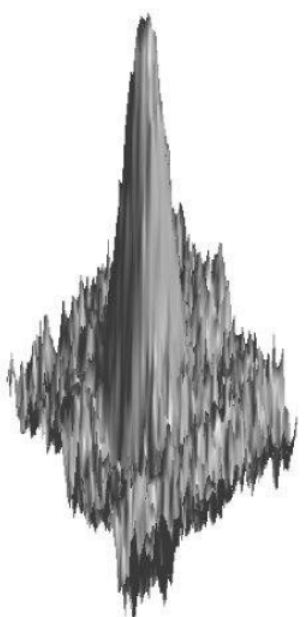

(a)

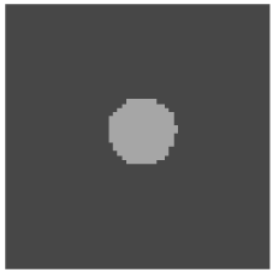

(b)

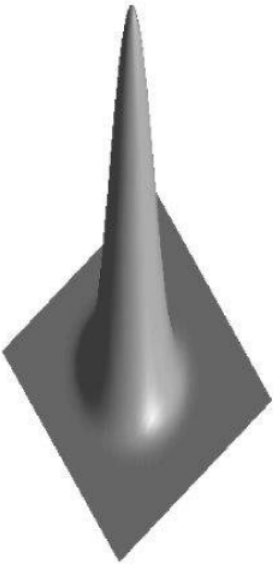

(c)

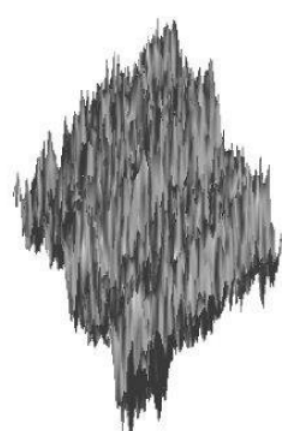

(d)

FIGURE 6. Comparison of measured and calculated thermal response of composite specimen. a. Thermal response at 1.0 second of flat bottom hole with a diameter of $0.319 \mathrm{~cm}, 0.99 \mathrm{~cm}$ below the front surface. b. Estimation of the shape of the top of the flat bottom hole based on variational method. c. Thermal response calculated based on shape estimated of the top of the flat bottom hole. d. Difference between the calculated thermal response and measured response.

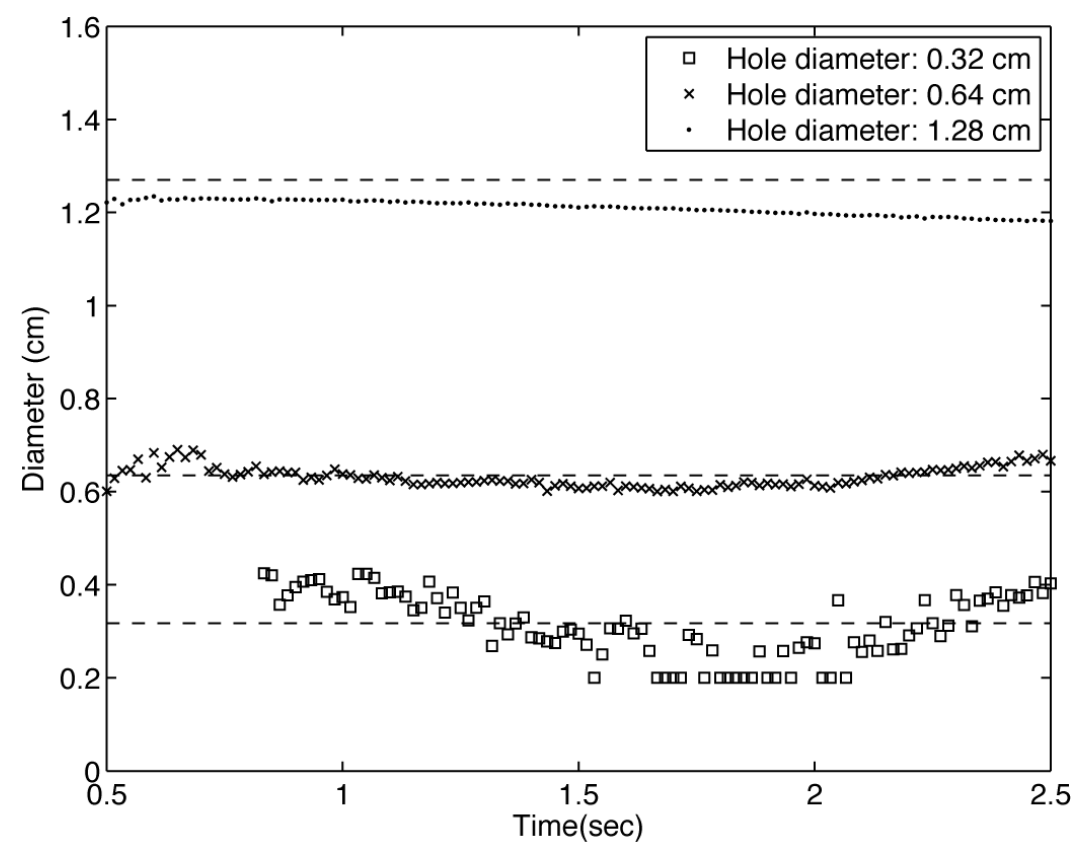

FIGURE 7. The variational method estimation of the diameter of the flat bottom holes with tops approximately $0.1 \mathrm{~cm}$ beneath the surface based on the reduction of images acquired at different times. A dashed line indicates the nominal diameter of the hole.

\section{CONCLUSIONS}

A method has been presented for determining the size of a flaw from the thermographic response using a variational approach to reduce the data. The technique was applied to thermographic data acquired on a flat bottom hole composite specimen, with flaws at different depths and diameters. From the estimated shapes of the holes, the hole diameters were found to be in good agreement with measurements of the hole diameters from computed tomography data on the specimen. The results were also compared with the estimated diameters from the same thermographic data using a 
method described by Almond and Lau[11]. There was good agreement between the estimated diameters from the two techniques for all but the smallest holes.

One of the advantages of the variational technique is that it is easily applied to shapes other than circular flat bottom holes. The shape obtained from the technique should be independent of the time step of the thermal data reduced. It is possible to reformulate the technique to reduce multiple time steps at the same time, to improve the shape estimation and estimate the depth of the flaw. Future efforts will focus on development of this formulation and testing the application of the technique to sizing of delaminations, which are of more interest in practical applications.

\section{REFERENCES}

1. D. Maillet, A. Houlbert, S. Didierjean, A. Lamine, and A. Degiovanni, Composites Science and Technology 47, 137-153 (1993).

2. D. Bates, G. Smith, D. Lu, and J. Hewitt, Composites Part B: Engineering 31, 175-185 (2000).

3. N. Avdelidis, B. Hawtin, and D. Almond, NDT\&E International 36, 433-439 (2003).

4. J. Schroedera, T. Ahmedb, B. Chaudhryb, and S. Shepard, Composites: Part A 33, 1511-1517 (2002).

5. N. P. Avdelidis, D. P. Almond, A. Dobbinson, B. C. Hawtin, C. Ibarra-Castanedo, and X. Maldague, Progress in Aerospace Sciences 40, 143-162 (2004).

6. W. P. Winfree, P. A. Howell, C. A. C. Leckey, and M. D. Rogge, "Improved sizing of impact damage in composites based on thermographic response," in Thermosense: Thermal Infrared Applications XXXV, edited by G. R. Stockton, and F. P. Colbert, 2013, vol. 8705 of Proc. SPIE.

7. S. M. Shepard, J. R. Lhota, B. A. Rubadeux, D. Wang, and T. Ahmed, Opt. Eng. 42, 1337-1342 (2003).

8. J. N. Zalamedaa, N. Rajic, and W. P. Winfree, "Thermographic determination of delamination depth in composites," in Thermosense XXV, edited by X. P. M. K. Elliot Cramer, 2003, vol. 5073 of Proc. SPIE, pp. 374-385.

9. Y. Plotnikov, and W. Winfree, "Temporal Treatment of a Thermal Response for Defect Depth Estimation," in Review of Progress in Quantitative NDE, edited by D. O. Thompson, and D. E. Chimenti, 2000, vol. 509 of AIP Conf. Proc., pp. 587-594.

10. W. P. Winfree, and J. N. Zalameda, "Thermographic determination of delamination depth in composites," in Thermosense XXV, edited by X. P. M. K. Elliot Cramer, 2003, vol. 5073 of Proc. SPIE, pp. 363-373.

11. D. P. Almond, and S. K. Lan, J.Phys.D: Appl.Phys. 27, 1063-69 (1994).

12. D. Almond, P. Delpech, M. Beheshtey, and P. Wen, "Quantitative Determination of Impact Damage and Other Defects in Carbon Fiber Composites by Transient Thermography," in Nondestructive Evaluation of Materials and Composites, edited by G. B. S.R. Docto, C.A. Lebowit, 1996, vol. 2944 of Proc. SPIE, pp. 256-264.

13. X. P. Maldague, Theory and Practice of Infrared Technology for Nondestructive Testing, John Wiley \& Sons, New York, New York, 2001, 1 edn., ISBN 0-471-18190-0.

14. D. J. Crowther, L. D. Favro, P. K. Kou, and R. L. Thomas, Journal of Applied Physics 74, 5828-5834 (1993).

15. L. D. Favro, D. J. Crowther, P. K. Kou, and R. L. Thomas, "Inverse Scattering of Pulsed Thermal Waves," in Advances in Signal Processing for Nondestructive Evaluation of Materials, edited by X.P.V.Malaque, 1994, vol. 262 of NATO Series E: Applied Science, pp. 187-191.

16. G. Aubert, and P. Kornprobst, Mathematical Problems in Image Processing, Partial Differential Equations and the Calculus of Variations, vol. 147 of Applied Mathematical Sciences, Springer, New York, New York, 2001, 1 edn., ISBN 0-387-95326-4.

17. T. F. Chan, and J. J. Shen, Image processing and Analysis: Variational, PDE, Wavelet and Stochastic Methods, SIAM, Philadelphia, Pennsylvania, 2005, 1 edn., ISBN 0-89871-589-X.

18. T. F. Chan, and L. A. Vese, IEEE Transactions on Image Processing 10, 266-277 (2001).

19. G. Aubert, and L. Vese, SIAM J. Numer. Anal. 5, 1948-1979 (1997). 